\title{
INVOLUTIONS ASSOGIATED WITH THE BURKHARDT CONFIGURATION IN [4]
}

\author{
A. F. HORADAM
}

1. Introduction. Horadam (11) has established the existence of a locus $L$ in [8] (projective 8-space) having order 45 and dimension 4 , which is invariant under a group of order $51840 \times 81$ (the Clifford similarity transform group $C T$ ). Associated with $C T$ are two other groups, the Clifford collineation group $C G$ of order 81, and the Clifford substitution group $C S$ of order 51840 . Furthermore, $C S$ may be regarded as either a subgroup of $C T$, or a symplectic group of index matrices of size 4 . Among the matrices of size 9 which perform the operations of $C T$, there is a set of 81 involutory, symmetric, orthogonal matrices $J W$. As collineation matrices in [8], these produce 81 pairs of invariant spaces 2 , $\Pi$ of dimensions 3 and 4 respectively. These [4]'s give rise to a configuration $\mathbf{G}$ invariant under the operations of $C T$, consisting of 360 points, 1080 lines, 120 Jacobian planes, and 81 [4]'s, and their various inter-relationships.

Familiarity with the theory and notation of (11) is assumed in this paper.

If a section of $L$ is taken by the $\Pi$-space whose determining equations are $x_{i j}=x_{2 i 2 j}(i, j=0,1,2)$, the resulting locus consists of just 45 points forming a Clifford-derived configuration identical with the Burkhardt configuration in [4], $\mathbf{B}$, which is associated with the rational Burkhardt quartic primal. Such points (nodes in B) have co-ordinates of two types, namely,

Type I: $\left(-2 \epsilon^{t} \epsilon^{u} \epsilon^{v} \epsilon^{w}\right)$ with $t+u+v+2 \equiv 0(\bmod 3)$.

Type II: $\left(. ; 1-\epsilon^{a}\right.$.. .).

Twenty-seven of these nodes belong to Type I and the remaining 18 to Type II. A general node of Type I will, for convenience, be labelled $P_{t u v w}$, or merely $P_{t}$, where there is no possible ambiguity, while the given node of Type II will be designated by $P_{a}$.

Comparison may be made between our Clifford-derived configuration, identical with $\mathbf{B}$, and the figure in [4] which Edge (6) has explained in detail. Based on $G F(3)$, this latter figure differs fundamentally from $\mathbf{B}$, but, at the same time, displays striking similarities.

2. Involutions concerning nodes and their Jordan primes. Invariance of $\mathbf{B}$ is preserved by the harmonic inversion, or projection $p(A)$, with respect to any node $A$ and its Jordan prime. This projection is the operation of the group $\frac{1}{2} C S$ (subgroup of $C S$ of index 2 and also the cubic surface (sub)group) which leaves invariant $A$ and the 12 nodes in the Jordan prime of $A$, and

Received November 26, 1957. 
which interchanges in pairs the two nodes, other than $A$, on each of the 16 $\kappa$-lines through $A$. Thus $p(A)$ is involutory. (Jordan primes in the $\Pi$ lie on $L$ and do not intersect any of the 81 solids $\Sigma$.) Operations relating to nodes $P_{t}$ and $P_{a}$ will usually be denoted by $p(t)$ and $p(a)$ respectively. Explicit matrix forms for the involutory projections $p(t)$ have been obtained in (11), wherein the two corresponding involutory matrices in $[8], p^{*}(t)$ and $p^{* *}(t)$ $=J p^{*}(t)$, which induce identical collineations in $\Pi$, and the associated involutory symplectic index matrices $\mathbf{p}(t)$ and $\mathbf{p}^{\prime}(t)=2 \mathbf{p}(t)$ are also found. (Starred notation used in (11) has been altered here for convenience.)

For the 18 Burkhardt nodes of Type II, the related matrices, which are not given in (11), are much less complicated. Since they fall into six similar triads, there is nothing lost in concentrating on only one triad, namely $P_{a}$ $(a=0,1,2)$. We deduce that

$$
\mathbf{p}(a)=\left[\begin{array}{ccccc}
1 & \cdot & \cdot & \cdot & \cdot \\
\cdot & \cdot & \epsilon^{2 a} & \cdot & \cdot \\
\cdot & \epsilon^{a} & \cdot & \cdot & \cdot \\
\cdot & \cdot & \cdot & 1 & \cdot \\
\cdot & \cdot & \cdot & \cdot & 1
\end{array}\right] .
$$

Correspondingly, in [8], $p^{*}(a)$ has only one non-zero element in each row and column, these being: unity in positions $\alpha_{00}{ }^{00}, \alpha_{11}{ }^{11}, \alpha_{21}{ }^{12}, \alpha_{12}{ }^{21}, \alpha_{22}{ }^{22} ; \epsilon^{a}$ in positions $\alpha_{10}{ }^{01}, \alpha_{20}{ }^{02}$; and $\epsilon^{2 a}$ in positions $\alpha_{10}{ }^{10}, \alpha_{02}{ }^{20}$. Of course, $p^{*}(a)=J p^{*}(a)$. Index matrices associated with these are

$$
\mathbf{p}(a)=\left[\begin{array}{rrrr}
\cdot & \cdot & 1 & 2 a \\
\cdot & \cdot & \cdot & 1 \\
1 & a & \cdot & \cdot \\
\cdot & 1 & \cdot & \cdot
\end{array}\right]
$$

of which the elements belong to the finite field $G F(3)$. The basic Clifford set is easily shown to be converted by $\mathbf{p}(0), \mathbf{p}(1), \mathbf{p}(2)$ into the Clifford sets

$$
\begin{array}{lllll}
W_{00}^{01}, & W_{01}^{01}, & W_{01}^{10}, & W_{11}^{10}, & W_{11}^{00}, \\
W_{00}^{01}, & W_{01}^{02}, & W_{01}^{11}, & W_{11}^{01}, & W_{11}^{21}, \\
W_{00}^{01}, & W_{01}^{00}, & W_{01}^{12}, & W_{11}^{22}, & W_{11}^{12},
\end{array}
$$

respectively, while each $\mathbf{p}^{\prime}(a)$ has the effect of doubling the indices throughout the set.

If the projection refers to the node $\left(. ; \ldots 1-\epsilon^{a}\right)$, then it is found that the corresponding index matrix is

$$
\left[\begin{array}{cccc}
2 & \cdot & \cdot & a \\
\cdot & 2 & \cdot & \cdot \\
\cdot & 2 a & 1 & 1 \\
\cdot & \cdot & \cdot & 1
\end{array}\right],
$$


a result used later in $\S 5$. Eight-dimensional forms and the corresponding reduced form in [4], together with the Clifford set arising from the basic Clifford set under the appropriate transformations, are quite easily established in a manner similar to that just stipulated.

Perhaps it is worth remarking that for any matrix $p(t)$ for which we have $\{p(t)\}^{2}=9 I$, each column of $p(t)-3 I$ yields the co-ordinates of the node $P_{t}$, whereas each row of $p(t)-3 I$ yields the prime co-ordinates of the Jordan prime of $P_{t}$. In the case of the matrices $p(a)$, the appropriate matrix whose columns and rows give rise to the node $P_{a}$ and its Jordan prime respectively, is $p(a)-I$, since $\{p(a)\}^{2}=I$.

Besides the 45 involutions (harmonic inversions) just enumerated, there are 270 other involutions associated with $\mathbf{B}$. These are analysed in $\S 5$. The aim of this paper is, specifically, to find matrix forms for these $45+270=315$ involutions, to discover their symplectic forms and their augmented forms as matrices of size 9 (also the effect of these on the basic Clifford set), and to relate the invariant spaces of these (collineation) matrices to the invariant configuration $\mathbf{C}$ we know to exist in [8]. Part of this objective has been achieved in the above section.

3. Invariant spaces of the $\mathbf{4 5}$ harmonic inversions. Next, we examine the invariant spaces in [8] generated by the 45 harmonic inversions. For the harmonic inversion $p(t)$, the invariant solid $\Delta_{t u v w}$, or simply $\Delta_{t}$ when there is no confusion, is found to be determined by the 4 points:

$\left(\begin{array}{ccccccccc}1 & \epsilon^{t} & \epsilon^{t} & \cdot & \cdot & \cdot & \cdot & \cdot & \cdot\end{array}\right)$

with $t, u, v, w=0,1,2$ and $t+u+v+w \equiv 0(\bmod 3)$. Each of these 4 points remains unchanged under the given collineation. Altogether, nine solids $\Delta$ pass through each of the points of (i).

Similarly, the invariant [4], $\Gamma_{t u v w}$, or $\Gamma_{t}$, is uniquely determined by four primes whose prime co-ordinates are like the set (i), except that the indices are everywhere doubled. By their nature, $p^{*}(t)$ and $p^{\prime *}(t)$ effect the same invariance of spaces. Nine $\Gamma$ lie in each prime.

Clearly, all the 26 solids $\Delta_{t}$ lie in the [4] II since, by (11) the 12 points in (i) are among the 40 points of $\Pi$ invariant under $J W$. Dually, all the 27 $\Gamma_{t}$ pass through the solid $\Sigma$ (corresponding to the II) determined by the four points $A_{i j}-A_{2 i 2 j}(i, j=0,1,2)$. As we know, $\Sigma$ lies on $L$ so that each $\Gamma_{t}$ cuts $L$ in the same solid $\Sigma$.

Furthermore, since the [4]'s II and a particular $\Gamma_{t}$ lie in [8], they must normally meet in a point. Now, by (ii), $\Pi$ may equally well be determined by the five points $A, B_{i}(i=1,2,3,4)$ from which we derive the point ( -2 $\left.\epsilon^{t} \epsilon^{t} \epsilon^{u} \epsilon^{v} \epsilon^{v} \epsilon^{u} \epsilon^{w} \epsilon^{v}\right)$ which, from our remarks about the primes determining 
$\Gamma_{t}$, obviously lies in the $\Gamma$. Of course, this common point is merely the node $P_{t}$ with which the harmonic inversion is associated. It does not belong to the configuration $\mathbf{G}$ in [8]. Thus, we have shown that the $27 \quad \Gamma_{t}$ constitute a family of [4]'s through $\Sigma$.

Dually, the solid $\Delta_{t}$, which lies entirely in $\Pi$, joins $\Sigma$ to a prime. In prime co-ordinates, this is $\left(-2 \epsilon^{t} \epsilon^{t} \epsilon^{u} \epsilon^{v} \epsilon^{v} \epsilon^{u} \epsilon^{w} \epsilon^{v}\right)$ and must not be confused with the prime $\left(-1 \epsilon^{t} \epsilon^{t} \epsilon^{u} \epsilon^{v} \epsilon^{w} \epsilon^{u} \epsilon^{w} \epsilon^{v}\right)$ whose [4] section by II produces the Jordan (polar) prime of the node $P_{t}$. (Actually, these two primes in [8] meet in a secundum through which the simplex prime $X_{00}=0$ passes.)

Systematising these results, we have, for the 27 nodes $P_{t}$ :

each $\Gamma$ through $\Sigma$ meets $\Pi$ in a node;

each $\Delta$ in $\Pi$ joins $\Sigma$ to a prime.

Irregularities occur in the case of the remaining 18 nodes $P_{a}$.

Taking our standard $P_{a}$, and considering both $p^{*}(a)$ and the associated matrix $p^{*}(a)=J p^{*}(a)$ which produce identical invariant spaces, we find that the [4] $\Gamma_{a}$ is determined by the four primes (in prime co-ordinates)

\begin{tabular}{|c|c|c|c|c|c|c|}
\hline 1 & & $\epsilon^{2 a}$ & . & . & & . \\
\hline . & 1 & . & 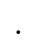 & . & $\epsilon^{2 a}$ & . \\
\hline . & . & . & 1 & . & . & . \\
\hline . & . & . & . & 1 & . & 1 \\
\hline
\end{tabular}

Alternatively, $\Gamma_{a}$ is determined by the five points

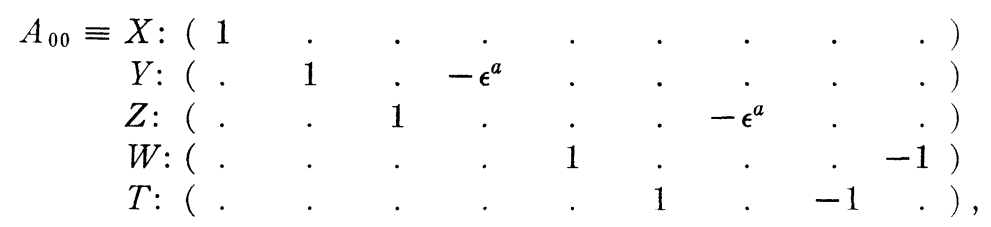

none of which belongs to the configuration $\mathbf{C}$, except $X$. Points $W, T$ and $Y-Z$ are contained in $\Sigma$, while points $X$ and $Y+Z$ belong to $\Pi$. Of course, $Y+Z$ is merely the [8] form of the node $P_{a}$.

Elements determining $\Delta_{a}$ are either

four points corresponding to the four defining primes of $\Gamma_{a}$, or five primes corresponding to the five defining points of $\Gamma_{a}$,

with the symbol $a$ replaced everywhere by $2 a$ in both cases. None of the four defining points of $\Delta_{a}$ belongs to $\mathbf{C}$.

So, for the $18 P_{a}$ we find that each $\Gamma_{a}$ meets $\Sigma$ in a plane (not a Jacobian plane) and $\Pi$ in a line (joining $P_{a}$ to $A_{00}$ ), that is, each $\Gamma_{a}$ has three points in $\Sigma$ and two points in $\Pi$, of which $A_{00}$ belongs to $\mathbf{C}$.

Similarly, by duality, each $\Delta_{a}$ lies in three primes of $I I$ and two primes of $\Sigma$, that is, each $\Delta_{a}$ is the intersection of $a$ [5] through $\Pi$ and $a$ [6] (secundum) 
through $\Sigma$, this secundum being the intersection of the prime dual of $P_{a}$ and the simplex prime $x_{00}=0$. Points of $\mathbf{C}$ are alien to $\Delta_{a}$ though the solid may be shown to possess three points of $\Sigma$ and one of $I$.

With regard to the matrix $p^{*}(a)$, it is worth noting that there are four possible arrangements of the four units, in pairs, all of which yield a matrix which leaves invariant the node and its corresponding Jordan prime, that is, they are involutory projections. However, as similarity matrices operating on a Clifford matrix $W$, they do not produce another $W$ (except in the case of the $p^{*}(a)$ chosen). That is, the other three possibilities are involutions which are not members of the Clifford group $C T$. Likewise for $p^{* *}(a)$. Such involutions do not belong to a group since the group multiplication property is absent.

4. Nodes in Jordan primes. Subsequently, it will be found useful to have the forms of the twelve points in a Jordan prime at out disposal. Now the polar (Jordan) prime of the node $P_{t}$ has prime co-ordinates $\left(-1 \epsilon^{2 t} \epsilon^{2 u} \epsilon^{2 v} \epsilon^{2 w}\right)$ in [4]. Suppose the node $P_{t^{\prime}}$ lies in this prime. To satisfy the incidence relation, we must have

$$
2+\epsilon^{2 t+t^{\prime}}+\epsilon^{2 u+u^{\prime}}+\epsilon^{2 v+v^{\prime}}+\epsilon^{2 w+w^{\prime}}=0,
$$

with the usual restrictions on the variables. That is, $2 t+t^{\prime}, 2 u+u^{\prime}, 2 v+v^{\prime}$, $2 w+w^{\prime}, \neq 0$. Therefore, $t^{\prime}=t+1, t+2$, and similarly for the other variables. Consequently, six nodes are given by

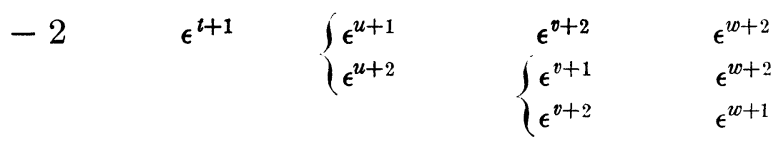

and

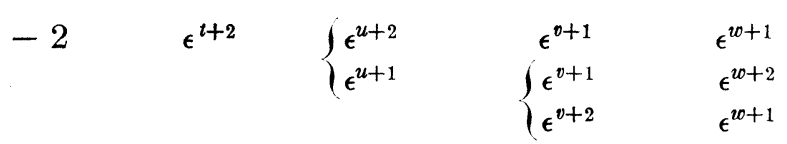

so that in the Jordan prime of a node $P_{t}$ we have just six nodes of Type I and, therefore, six of Type II.

Regarding nodes $P_{a}$, we observe that the corresponding Jordan prime has prime co-ordinates $\left(\cdot 1-\epsilon^{2 a} ..\right)$ in [4], so that only three nodes of Type II are contained therein, namely, the nodes $\left(\cdot .1-\epsilon^{a}\right)$. Accordingly, there must be nine nodes of Type $I$ in it.

5. Involutions concerning Jordan pentahedra. Apart from the 45 involutions which leave invariant a node and its Jordan prime and interchange the nodes on each $\kappa$-line through the given node, there is another set of involutory operations for which we can find matrix forms. Following Baker (1), Todd (12) has shown that such operations of period 2 form a conjugate set, and are the products of pairs of projections. 
Now, it is known (12) that the twelve nodes of a Jordan prime fall into three sets of 4 , forming a triad of desmic tetrahedra, any two of which are in perspective from each vertex of the third. Such a tetrahedron, together with the pole of the Jordan prime, forms a Jordan pentahedron. Otherwise stated, a Jordan pentahedron is a symmetrical set of five nodes such that the polar prime of any one contains the other four, and the join of any two nodes is an $e$-line and the plane containing any three nodes is an $f$-plane, that is, a plane containing three nodes whose joins, in pairs, are $e$-lines. (By an $e$-line we mean a line joining a node $A$ to a node $B$ in the Jordan prime of $A$, and containing no other point.) There are 27 such Jordan pentahedra in $\mathbf{B}$ (three through a given node), as well as $270 f$-planes, $270 e$-lines, and $240 \kappa$-lines.

Suppose $A B$ is an $e$-line. Then

$$
p(A) p(B)=p(B) p(A)
$$

so that from each $e$-line there arises one commutative operation $p(A) p(B)$. Todd remarks that $p(A)$ and $p(B)$ generate an Abelian group of order 4 , direct product of the cyclic groups generated by the projections $p(A) p(B)$. Further, $p(A) p(B)$ is an axial homology having for invariant spaces the $e$-line $A B$ and the opposite plane ( $f$-plane) $C D E$ of the Jordan pentahedron having $A B$ for an edge. Consequently, $p(A) p(B)$ leaves invariant the nodes $A, B$, $C, D, E$ and just these.

Here we may interpolate the result, stated in Todd, that if $A B$ is a $\kappa$-line with $C$ the third point on it, then

$$
p(A) p(B)=p(B) p(C)=p(C) p(A),
$$

so that, under these circumstances, $p(A) p(B)$ has period 3 . In addition, Todd remarks that, if $A B C D E$ is a Jordan pentahedron, then

$$
p(A) p(B) p(C) p(D) p(E)=1 \text {. }
$$

Both these results are easy to verify in terms of our matrices.

Because of the different co-ordinate forms for the nodes $P_{t}$ and $P_{a}$, the matrices for $p(A) p(B)$ will have varying forms. Having regard to the comments in $\S 4$ about the type of a node in a Jordan prime, we realise that the 270 involutions may be classified in the following way:

For $p(A) p(B)$ of Type $\mathrm{I} \times$ Type $\mathrm{I}$, there are $27 \times 6=162$ possibilities,

For $p(A) p(B)$ of Type I $\times$ Type II, there are $27 \times 6+18 \times 9=324$ possibilities,

For $p(A) p(B)$ of Type II $\times$ Type II, there are $18 \times 3=54$ possibilities.

However, since each $e$-line occurs twice in the classification, on account of the commutativity property, we find that the numbers of involutions are $81,162,27$ (totalling 270).

Attention is next focused on the set of 81 involutions. On multiplying together the matrices for the projections of two general nodes of Type I, 


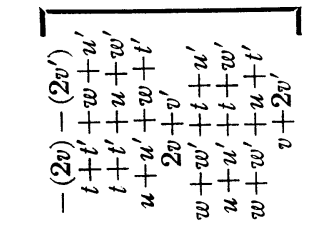

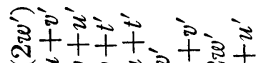

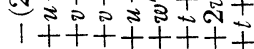

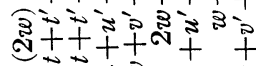

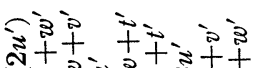

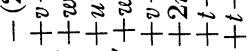

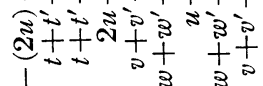

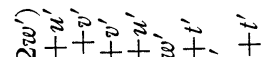

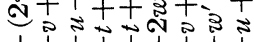
$1++++++++$

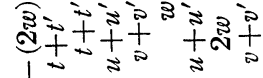

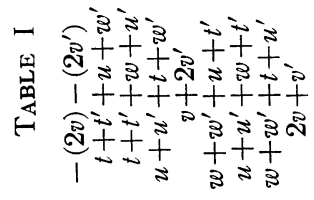

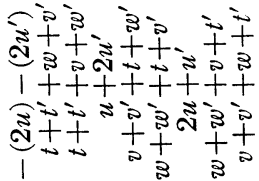

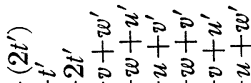

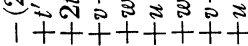

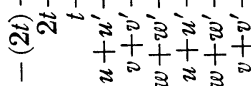

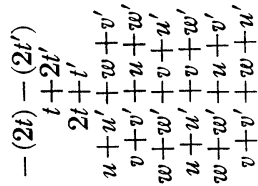

สิริ)

011111111

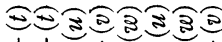

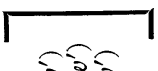

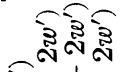

3+t+

๙ูㅊㅊㅇㅡ

$1+++1$

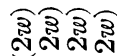

$\mathrm{N}+++$

$1=3$

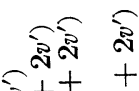

ติำ

लㅊำอ

$1++1+$

ลิลลิล ลิ

N++ +

$1 \approx 3$

ลิ ลิ๋

in +

लูป

$1+1++$

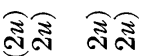

N+ +

$1=23$

สิธิธี

ล ++

ผิองละ

$11+++$

ลิ สิธิธิธิ

ก +++

롤ㅈㅈㅣ

ฮิฐิ

01111

อ조옹

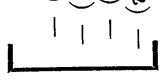

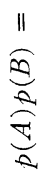

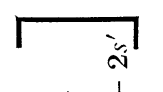

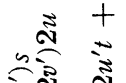

กิ่

$++$

ว

$+++$

กิ

$++$

쿠류

ล

$+$

$\approx$ ลั

a.

$+\stackrel{+}{+2}$

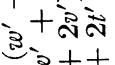

$++3$

ลิ ลิ

3
$+^{3}+3$
$4^{2}+9$

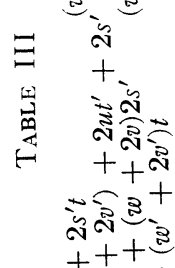

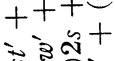

ลิล

$++$

궁조요

ลั

$\pm \overline{\overline{\mathrm{N}}}$

กิ ลิก

$+++$

ลิ่ง

$+++\frac{a}{2}$

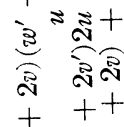

洨

$\|$

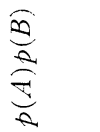

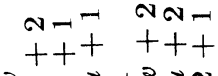

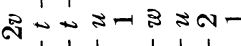

$++++++$

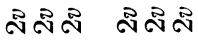

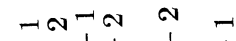

$++++t$

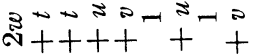

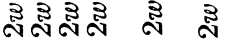

$\overrightarrow{+}+\overrightarrow{+}+$

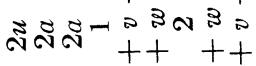

ลี่ีี ลีสี

$\mathrm{N}-\overrightarrow{+}+\overrightarrow{+}$
++

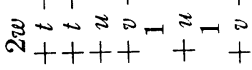

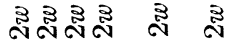

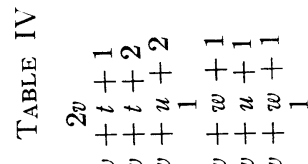

ลิลัก ลำล

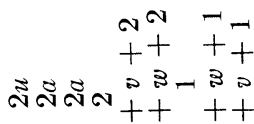

ลีกี สีสี

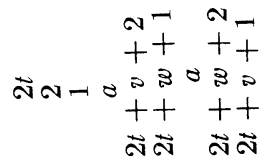

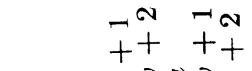

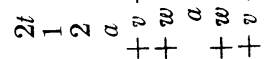

ลี สี

$0 \sim m a 53 \approx 3=$ 
say, $A \equiv P_{t}$ and $B \equiv P_{t^{\prime}}$, we find, on simplifying by means of (ii) where necessary, and using the convention of (11), that the matrix form of $p^{*}(A)$ $p^{*}(B)$ is as shown in Table I. Actually, the convention has been slightly extended, to allow us to interpret (say) $-(2 t)-\left(2 t^{\prime}\right)$ as $-\epsilon^{2 t}-\epsilon^{2 t^{\prime}}$, that is, only the portion in brackets is a power of $\epsilon$ in cases like this.

Interchanging in pairs rows 2 and 3,4 and 7,5 and 9 , and 6 and 8 , that is, operating on this matrix by $J$, we obtain the companion matrix $\left\{p^{*}(A) p^{*}(B)\right\}^{\prime}$ $=p^{*}(B) p^{*}(A)$ which induces the same collineation as the above matrix. Further, $p^{*}(B) p^{*}(A)$ is the inverse of $p^{*}(A) p^{*}(B)$. From these facts, we deduce that

$$
\left\{p^{*}(A) p^{*}(B)\right\}^{2}=\left\{p^{*}(B) p^{*}(A)\right\}^{2}=J
$$

in [8], that is, the operation $p^{*}(A) p^{*}(B)$ in [8] is not involutory, but has period 4. On account of the relationship $p^{*}(A)=J p^{*}(A)$, we have

$$
p^{*}(A) p^{*}(B)=J p^{*}(A) p^{*}(B)=p^{*}(B) p^{*}(A) .
$$

Making the necessary adjustments for the form of the projection-product in [4], we have, using our convention again, the equation in Table II. As this is symmetrical in dashed and undashed letters, we have

$$
p(A) p(B)=p(B) p(A)
$$

as we expect, and, of course, $\{p(A) p(B)\}^{2}=I$, that is, the operation is involutory.

Finally, the general symplectic matrix is shown in Table III where $s=(w+2 v)^{2}+2 u t+2, s^{\prime}=\left(w^{\prime}+2 v^{\prime}\right)^{2}+2 u^{\prime} t^{\prime}+2$, and the arithmetic is reduced mod 3 .

Squaring this matrix always gives $2 I$ so that its period is 4 . Calculation of the square is complicated and tedious.

In particular, consider the nodes $A \equiv P_{0000}$ and $B \equiv P_{1122}$. On calculation, the reduced matrix form for $p(A) p(B)$ is found to be

$$
\left[\begin{array}{ccccc}
1 & 2 \epsilon & 2 \epsilon & 2 \epsilon^{2} & 2 \epsilon^{2} \\
\epsilon^{2} & -1 & 2 & -\epsilon & -\epsilon \\
\epsilon^{2} & 2 & -1 & -\epsilon & -\epsilon \\
\epsilon & -\epsilon^{2} & -\epsilon^{2} & -1 & 2 \\
\epsilon & -\epsilon^{2} & -\epsilon^{2} & 2 & -1
\end{array}\right]
$$

which leaves invariant the points $A, B$ of the $e$-line and the points $C \equiv P_{0}$, $D \equiv(. .1-1)$ and $E \equiv P_{2211}$ of the $f$-plane in the Jordan pentahedron. Additionally, in its [8] form, $p^{*}(A) p^{*}(B)$, as a member of $C T$, transforms the basic Clifford set into the Clifford set

$$
W_{20}^{10} \quad W_{10}^{00}, \quad W_{22}^{21}, \quad W_{21}^{20}, \quad W_{22}^{22} .
$$

Correspondingly, the index matrix is 


$$
\left[\begin{array}{llll}
1 & 2 & \cdot & \cdot \\
2 & 2 & \cdot & \cdot \\
\cdot & \cdot & 1 & 2 \\
\cdot & \cdot & 2 & 2
\end{array}\right]
$$

of which the square is $2 I$.

Next, we examine the invariant spaces associated with the operation $p^{*}(A) p^{*}(B)$, whose period is 4 . Direct calculation shows that the point

$$
\left(1,-\left(\epsilon^{t}+\epsilon^{t^{\prime}}\right),-\left(\epsilon^{t}+\epsilon^{t^{\prime}}\right) \ldots\right)
$$

is transformed into the point whose first three co-ordinates are $3 \ldots$, and whose succeeding three co-ordinates are

$$
\begin{aligned}
& -\left\{2\left(\epsilon^{u}+\epsilon^{u^{\prime}}\right)+\epsilon^{u^{\prime}+2 l^{\prime}+t}+\epsilon^{u+2 t+t^{\prime}}\right\} \\
& -\left\{2\left(\epsilon^{v}+\epsilon^{v^{\prime}}\right)+\epsilon^{v^{\prime}+2 t^{\prime}+t}+\epsilon^{v+2 t+t^{\prime}}\right\} \\
& -\left\{2\left(\epsilon^{w}+\epsilon^{\omega^{\prime}}\right)+\epsilon^{\omega^{\prime}+2 t^{\prime}+t}+\epsilon^{i+2 t+t^{\prime}}\right\}
\end{aligned}
$$

with corresponding co-ordinates appropriately situated in the last three positions. Note that the co-ordinates are symmetrical in dashed and undashed symbols so that $p^{*}(A) p^{*}(B)$ and $p^{*}(B) p^{*}(A)$ produce the same invariant solid. Similar forms apply for the transformations of the $u$-, $v$-, and $w$-co-ordinates. Only one of the three pairs of co-ordinates in positions 4 and 7, 5 and 9 , and 6 and 8 , is non-zero, a fact we now proceed to establish.

Firstly, suppose that all three pairs are simultaneously non-zero. Then

$$
\begin{aligned}
u^{\prime}+2 t^{\prime}+t+u+2 t+t^{\prime} & =u+u^{\prime} \\
v^{\prime}+2 t^{\prime}+t+v+2 t+t^{\prime} & =v+v^{\prime} \\
w^{\prime}+2 t^{\prime}+t+w+2 t+t^{\prime} & =w+w^{\prime}
\end{aligned}
$$

which imply (say)

$$
\begin{aligned}
u^{\prime}+2 t^{\prime}+t & =u \text { or } u^{\prime} \\
v^{\prime}+2 t^{\prime}+t & =v \text { or } v^{\prime} \\
w^{\prime}+2 t^{\prime}+t & =w \text { or } w^{\prime}
\end{aligned}
$$

(Calculations hereunder are taken modulo 3.) Let $u^{\prime}+2 t^{\prime}+t=u^{\prime}$. Therefore, $2 t^{\prime}+t=0$ so that $t^{\prime}=t$ which is clearly impossible since one point lies in the Jordan prime of the other.

Consequently, take $u^{\prime}+2 t^{\prime}+t=u$ with similar selections in (b) and (c). Combining these results, we have $2 u+u^{\prime}=2 v+v^{\prime}=2 w+w^{\prime}=2 t+t^{\prime}$ which is obviously invalid from the incidence relation (ii).

Assume next that all three pairs vanish together. Then

$$
\begin{aligned}
u^{\prime}+2 t^{\prime}+t & =u+2 t+t^{\prime}=2\left(u+u^{\prime}\right) \\
v^{\prime}+2 t^{\prime}+t & =v+2 t+t^{\prime}=2\left(v+v^{\prime}\right) \\
w^{\prime}+2 t^{\prime}+t & =w+2 t+t^{\prime}=2\left(w+w^{\prime}\right)
\end{aligned}
$$

whence 


$$
2 u+u^{\prime}=2 v+v^{\prime}=2 w+w^{\prime}=t+2 t^{\prime}
$$

which, by virtue of (ii), is essentially inadmissible.

Accordingly, the three pairs cannot be either simultaneously zero or simultaneously non-zero. Suppose, then, that the $u$-co-ordinates vanish but not the $v$-co-ordinates. This assumption involves $2 u+u^{\prime}=t+2 t^{\prime}=2\left(2 t+t^{\prime}\right)$ (as above) and $v^{\prime}+2 t^{\prime}+t=v$, that is, $v+2 v^{\prime}=t+2 t^{\prime}$. Since, from (ii), two powers of $\epsilon$ are twice the other two, we must have

$$
\begin{aligned}
2 w+w^{\prime} & =2\left(2 v+v^{\prime}\right) \\
& =t+2 t^{\prime}
\end{aligned}
$$

which means that the w-co-ordinate also vanishes. Analogous reasoning for the vanishing of the $v$-co-ordinates or of the $w$-co-ordinates obviously applies. Of the three pairs of co-ordinates, therefore, only one is non-zero.

To illustrate the theory, choose the nodes $P_{0120}$ and $P_{1002}$. We find that $p(0120) p(1002)$ leaves invariant the solid $\Delta_{2211}$. However, this projectionproduct differs from the projection $p(2211)$, which leaves $\Delta_{2211}$ pointwise invariant, in that it effects the substitution utwr on the co-ordinates $t u v w$ as specified in (i). The same solid $\Delta_{2211}$ may be shown to arise invariantly from the projection-products $p(0120) p(1002)$ and $p(0102) p(1020)$ with substitutions $v w t u$ and $w v u t$ respectively of $t u v w$. To see this in (say) the first case, we take the vanishing of the $u$-and $w$-co-ordinates with the equation

$$
2\left(\epsilon^{v}+\epsilon^{v^{\prime}}\right)+\epsilon^{v^{\prime}+2 t^{\prime}+t}+\epsilon^{t+2 t+t^{\prime}}=-3 \epsilon \quad\left(t=0, t^{\prime}=1\right)
$$

and solve these equations.

In the manner indicated above, it is verifiable that the 81 invariant solids formed from the projection-products $p(t) p\left(t^{\prime}\right)$ are merely the 27 solids $\Delta$, each occurring three times. Generally, to the projection $p(T)$ correspond three projection-products $p(t) p(2 t+2 T)$. This is seen by letting $t^{\prime}=t+\alpha$ in the projection-product corresponding to the projection $p(T)$. Comparing the $t$-co-ordinates in the two matrices, we have

$$
\begin{aligned}
\epsilon^{2 T} & =-\left(\epsilon^{2 t}+\epsilon^{2 \iota^{\prime}}\right) & \left(t^{\prime}=t+\alpha\right) \\
& =\epsilon^{2 t+\alpha} &
\end{aligned}
$$

so that $\alpha=2 T+t$. But $\alpha=t^{\prime}+2 t$, so that $t^{\prime}+2 t=2 T+t$ whence $t^{\prime}=2 t+2 T$.

Similarly for the $U_{-}, V$-, and $W$-co-ordinates. There does not appear to be any general matrix connection between $p(T)$ and $p(t) p(2 t+2 T)$, though in particular cases a simple relation may occur. For instance, $p(2211)=$ $K p(0000) p(1122)$ where $K$ corresponds to the substitution (174285 396) and so $K^{2}=J$.

Likewise, the 81 [4]'s $\Gamma_{t}$ are merely the original 27 , each occurring three times. 
Turning now to projection-products of Type I $\times$ Type II, we find, on using the standard projection $p(a)$ of Type II, that $p(t) p(a)$ yields the matrix in Table IV. When squared, this matrix gives $J$, showing that it is of period 4. Operating on this matrix by $J$, we obtain the companion matrix which produces the same invariance of spaces in [8]. Also

$$
p^{*}(a) p^{*}(t)=J p^{*}(t) p^{*}(a)=p^{*}(t) p^{*}(a) .
$$

In [4], the above matrix becomes

$$
p(t) p(a)=\left[\begin{array}{ccccc}
0 & 2(2 t) & 2(2 u) & 2(2 v) & 2(2 w) \\
t & -(0) & 2(2 a) & -(2 v+t) & -(2 w+\mathrm{t}) \\
u & 2(a) & -(0) & -(2 v+u) & -(2 w+u) \\
v & -(2 t+v) & -(2 u+v) & 2(0) & -(2 w+v) \\
w & -(2 t+w) & -(2 u+w) & -(2 v+w) & 2(0)
\end{array}\right]=p(a) p(t)
$$

of which the square is $I$.

For the projection-product to refer to an $e$-line, the incidence relation must be

$$
\epsilon^{t}=\epsilon^{u+2 a} \text {, that is, } t=u+2 a
$$

a fact used in the matrix calculation above and again in the determination of the invariant solid hereunder.

It is easy to verify that, as a collineation matrix, $p(t) p(a)$ has the effect of interchanging the $t$ - and $u$-co-ordinates of the solid $\Delta$, whilst leaving unaltered the $v$ - and $w$-co-ordinates, that is, of performing the substitution utvw on tuvw. Clearly, the same $\Delta$ arises from the substitutions vutw, wuvt, tvuw, twru, and tuwv. Hence, each $\Delta$ is repeated six times, thus absorbing the $27 \times 6=162$ matrices of this kind. It is noted that the substitution utvw corresponds to the node having only $t$ - and $u$-co-ordinates non-zero, viz., the node $P_{a}$. Likewise for the other possibilities.

Similarly, each of the 27 [4]'s $\Gamma$ occurs six times as an invariant space.

Writing $M=w+2 v$, we have, on multiplying, that

$$
\mathbf{p}(a) \mathbf{p}(t)=\left[\begin{array}{llll}
t & a t+s & M & 2 a M \\
1 & 2 t & . & M \\
M & a M & 2 u & a u+2 s \\
\cdot & M & 2 & u
\end{array}\right]
$$

Squaring, we get $\{\mathbf{p}(a) \mathbf{p}(t)\}^{2}=2\left(M^{2}+1\right) \mathbf{I}$, where $M^{2}=0,1$. Thus, $p(a) p(t)$ is involutory if $M^{2}=1$, that is, if $M=1,2$, but is of period 4 if $M=0$, that is, if $w=v$.

Illustrating the above in the case $A$ and $C$ (given earlier), we have 


$$
p(A) p(C)=\left[\begin{array}{rrrrr}
1 & 2 & 2 & 2 & 2 \\
1 & -1 & 2 & -1 & -1 \\
1 & 2 & -1 & -1 & -1 \\
1 & -1 & -1 & 2 & -1 \\
1 & -1 & -1 & -1 & 2
\end{array}\right]
$$

which leaves invariant the nodes $A, C$ of the $e$-line and the points $B, D, E$ of the $f$-plane in the Jordan pentahedron. As a (non-involutory) matrix of $C T, p^{*}(A) p^{*}(C)$ may be shown to transform the basic Clifford set into the Clifford set

$$
W_{10}^{00}, \quad W_{10}^{20}, \quad W_{02}^{20}, \quad W_{02}^{21}, \quad W_{00}^{21}
$$

and the index matrix performing this operation is

$$
\left[\begin{array}{cccc}
\cdot & 2 & \cdot & \cdot \\
1 & \cdot & \cdot & \cdot \\
\cdot & \cdot & \cdot & 1 \\
\cdot & \cdot & 2 & \cdot
\end{array}\right]
$$

of period 4. Regarded as a collineation matrix in [8], $p^{*}(A) p^{*}(C)$ is found to leave the solid $\Delta_{0000}$ invariant. Incidentally, $p(0000)=\lambda p^{*}(A) p^{*}(C)$ where $\lambda$ corresponds to the substitution (174 396285 ) and thus has order 2 .

Epitomizing, we see that the effect of the $81+162=243$ projectionproducts associated with a projection of Type I, as collineation matrices, is to reproduce each of the 27 solids $\Delta$, and each of the [4]'s $\Gamma$ in the $3+6=9$ ways indicated. (These 9 substitutions do not form a group.) So the 243 projection-products make no effective contribution to the sets of invariant spaces.

Finally, consider the last category of projection-products. There are 3 sets of $9 e$-lines constituting the $27 e$-lines, to which these refer, namely, the $e$-lines joining

$$
\begin{aligned}
& \text { points (. } \left.\begin{array}{lllllllll} 
& 1 & - & \epsilon^{a} & \text {. } & \text {. } & \text { ) to points (. . . . } & 1 & -\epsilon^{b}
\end{array}\right)
\end{aligned}
$$

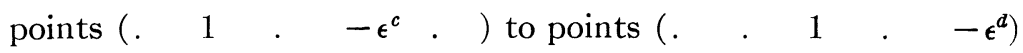

$$
\begin{aligned}
& \text { points (. } \left.1 . \quad . \quad-\epsilon^{e}\right) \text { to points (. . } 1-\epsilon^{\jmath} \quad . \quad \text { ), } \\
& a . ., f=0,1,2 \text {. }
\end{aligned}
$$

We find that $p^{*}(a) p^{*}(b)$ has only one non-zero element in each row and column, these being unity in position $\alpha_{00}{ }^{00}, \epsilon^{2 a}$ in positions $\alpha_{10}{ }^{01}$ and $\alpha_{20}{ }^{02}$, $\epsilon^{a}$ in $\alpha_{01}{ }^{10}$ and $\alpha_{02}{ }^{20}, \epsilon^{b}$ in $\alpha_{11}{ }^{12}$ and $\alpha_{22}{ }^{21}$, and $\epsilon^{2 b}$ in $\alpha_{21}{ }^{11}$ and $\alpha_{12}{ }^{22}$. Also,

$$
p^{*}(a) p^{*}(b)=J p^{*}(b) p^{*}(a) .
$$

The reduced form in [4] is 


$$
p(a) p(b)=\left[\begin{array}{ccccc}
1 & \cdot & \cdot & \cdot & \cdot \\
\cdot & \cdot & 2 a & \cdot & \cdot \\
\cdot & a & \cdot & \cdot & \cdot \\
\cdot & \cdot & \cdot & \cdot & 2 b \\
\cdot & \cdot & \cdot & b & \cdot
\end{array}\right],
$$

of which the square is $2 I$ so that $p(a) p(b)$ has period 4 . Using the symplectic form of $p(b)$ given in $\S 2$, we have, on multiplication,

$$
p(a) p(b)=\left[\begin{array}{cccc}
\cdot & 2 b & 1 & 2 a \\
\cdot & \cdot & \cdot & 1 \\
2 & 2 a & \cdot & b \\
\cdot & 2 & \cdot & \cdot
\end{array}\right]
$$

whose period is 4 .

In particular, if $a=2, b=0$, we may readily verify that $p^{*}(2) p^{*}(0)$ converts the basic Clifford set into the Clifford set

$$
W_{00}^{02}, \quad W_{02}^{00}, \quad W_{02}^{11}, \quad W_{12}^{21}, \quad W_{12}^{11},
$$

and the symplectic matrix performing this function is

$$
\left[\begin{array}{cccc}
\cdot & \cdot & 1 & 1 \\
\cdot & \cdot & \cdot & 1 \\
2 & 1 & \cdot & \cdot \\
\cdot & 2 & \cdot & \cdot
\end{array}\right]
$$

In [4], $p(2) p(0)$ leaves invariant the points $D$ (used earlier) and $F \equiv P_{2}$ of an $e$-line and also the points $G \equiv P_{2100}, H \equiv P_{1011}, I \equiv P_{0222}$ of the $f$-plane in the Jordan pentahedron.

Under the collineation $p^{*}(a) p^{*}(b)$ in [8], the solid $\Delta_{a b}$ determined by the 4 points

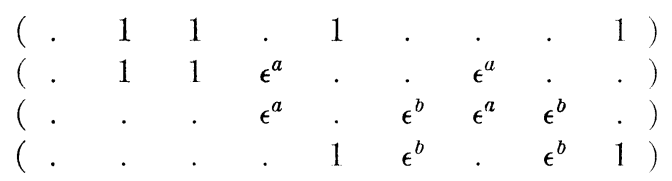

has the second and fourth points left invariant, while the first and third are interchanged. (None of these points belongs to the configuration $\mathbf{G}$ ).

Observation reveals that, as $a, b$ vary within their limits, the 10 points determining the $9 \Delta_{a b}$ lie wholly in $\Pi$. Moreover, consideration of the solids $\Delta_{c d}, \Delta_{e f}$ shows that, in addition to these 10 points, two other points, viz.,

$$
\left(\begin{array}{llllll} 
& 1 & 1 & \left.\cdot \epsilon^{g} . . . \epsilon^{g}\right) & (g=1,2)
\end{array}\right.
$$

will arise from the collineations $p^{*}(c) p^{*}(d)$ and $p^{*}(e) p^{*}(f)$. Just these 12 points of $\Pi$, but not in $\mathbf{C}$, thus suffice to define the 27 solids $\Delta_{a b}, \Delta_{c d}, \Delta_{e r}$. 
(Compare these with the twelve points of $\Pi$ in $\mathbf{C}$ - the set of points (ii) which suffice to determine the $27 \Delta_{t}$.) But these twelve points of $\Pi$ also manifestly lie in the simplex prime $x_{00}=0$ through $\Sigma$. Therefore, all 27 $\Delta_{a b}, \Delta_{c d}, \Delta_{e f}$ coalesce into one solid, viz., the solid of intersection of $\Pi$ and $x_{00}=0$. I find this an unexpected, but satisfying, simplification. Knowing, however, the perverse forms of matrix operators like $p(a)$ and remembering the importance of our basic spaces, perhaps we should not be too surprised at such eccentricities of behaviour.

Dually, the $27 \Gamma_{a b}, \Gamma_{c d}, \Gamma_{e f}$ are united as one [4], joining $A_{00}$ to $\Sigma$.

So the solid, $\Pi \cap x_{00}=0$, and the [4], $\Sigma+A_{00}$, are the invariant spaces of the 27 Type II $\times$ Type II operations.

A diagram will serve to emphasize the aesthetic simplicity of the invariant spaces we have been investigating.

6. Summary. Explicit matrix forms for the $45+270=315$ involutory operations used by Todd in connection with the configuration $\mathbf{B}$ have now been found. Extensions of these matrices to [8] and to the symplectic forms have been accomplished. As members of $C T$, the augmented matrices, numbering $315 \times 2=630$, are proved to have period 4 in $270 \times 2=540$ cases, and to be involutory in the remaining $45 \times 2=90$ cases. Symplectic matrices, 315 in all, are generally of period 4 , except the set of 45 , which are involutory, and those matrices like $p(a) p(t)$ for which $w=v$, which are also involutory. Moreover, the invariant spaces of the collineation matrices in [8] have been found and related to the configuration $\mathbf{C}$.

Geometrically, the results may be summed up in the following manner. As collineation matrices in [8], the 630 members of $C T$ leave invariant 315 solids $\Delta$ and 315 [4]'s $\Gamma$. Of these, only $46 \Delta$ and $46 \Gamma$ are distinct. Regarding the $\Delta$, we have:

$27 \Delta_{t}$ lie entirely in $\Pi$, being determined completely by twelve points of $\mathbf{C}$, and each lies in a prime through $\Sigma$;

$18 \Delta_{a}$ each of which lies in three primes through $\Pi$ and in two primes through $\Sigma$, and contains no points of $\mathbf{G}$;

$1 \Delta_{a b} \equiv \Pi \cap x_{00}=0$, containing no points of $\mathbf{C}$.

And dually there exist:

$27 \Gamma_{t}$ containing $\Sigma$ entirely, each meeting $\Pi$ in a Burkhardt node;

$18 \Gamma_{a}$ each meeting $\Sigma$ in a plane and $\Pi$ in a line, such that the simplex vertex $A_{00}$ is a common point of these eighteen lines;

$1 \Gamma_{a b} \equiv \Sigma+A_{00}$.

Intersection relations amongst the various $\Delta$ and $\Gamma$ may be obtained, but this objective is beyond the purpose of this paper. Details of the intersections of the invariant spaces with the locus $L$ and the configuration $\mathbf{B}$ may safely be left to the reader's curiosity.

The results embodied in the above summary apply to the [4] II given by $x_{i j}=x_{2 i 2 j}(i, j=0,1,2)$ and its dual space, the solid $\Sigma$ defined by $A_{i j}-A_{2 i 2 j}$ 
$(i, j=0,1,2)$. Obviously, they apply equally well in modified form to the other II- and $\Sigma$ - spaces whose invariance is preserved by the collineation matrices $J W$.

It is hoped that, in another paper, discussion of the complete set of involutions of the Clifford group $C T$ will be forthcoming. The set of $81 \mathrm{JW}$ and $90 p^{*}\left(54 p^{*}(t)\right.$ and $\left.36 p^{*}(a)\right)$ clearly do not form a group, as, for instance, the product of two $J W$ matrices yields merely a $W$. Also, the product of two $p^{* \prime} s$ is a matrix of period 4 , as we have shown.

7. Finale. Quite recently, some interesting and important articles have appeared relative to the group $C S$ of order 51840 which is known (11) to be the factor group $C T / C G$, and to the group $\frac{1}{2} C S$ of order 25920 .

Structural properties of $C S$ have, within the past few years, been investigated by Edge $(7 ; 8)$. In the former, he shows that this group has a representation by orthogonal matrices, of size 5 and determinant +1 , over $G F(3)$.

Dieudonné (4) has dealt with geometrical properties associated with two groups of order 25920 . These groups, the projective groups corresponding to the symplectic and unitary groups $S p_{4}\left(F_{3}\right)$ and $U_{4}+\left(F_{4}\right)$ respectively (in Dieudonné's notation), have been proved isomorphic by Dickson (3). Elsewhere, Dieudonné (5) discusses the automorphisms of these groups.

By (11), the generators $\mathbf{Q}$ and $\mathbf{D}$ of $\frac{1}{2} C S$ have periods 5 and 3 with defining relation $(\mathbf{Q D})^{5}=\mathbf{I}$. Compare these with the generators $S$ and $T$ of Brahana (2), which have periods 5 and 2 respectively, with $S T$ and $S^{2} T$ of periods 12 and 9 respectively. Frame (9) in examining an abstractly identical group, obtains two generators similar to Brahana's generators.

Finally, $C S$, the group of automorphisms of the 27 lines of a cubic surface is, Frame (10), the subgroup of the group of order $51840 \times 28=1451520$ of the automorphisms of the 28 bitangents to a plane quartic curve, which leaves one bitangent fixed. 


\section{REFERENCES}

1. H. F. Baker, A locus with 25920 linear self-transformations, Cambridge Tracts, 39 (1946).

2. H. R. Brahana, Pairs of generators of the known simple groups whose orders are less than one million, Ann. Math., 31 (1930), 529-49.

3. L. E. Dickson, Linear Groups with an Exposition of the Galois Field Theory (Leipzig, 1901).

4. J. Dieudonné, Les isomorphismes exceptionnels entre les groupes classiques finis, Can. J. Math., 6 (1954), 305-15.

5. - On the automorphisms of the classical groups, Mem. Amer. Math. Soc., 2 (1951).

6. W. L. Edge, Line geometry in three dimensions over $G F(3)$ and the allied geometry of quadrics in four and five dimensions, Proc. Roy. Soc. A, 228 (1955), 129-46.

7. - - The conjugate classes of the cubic surface group in an orthogonal representation, Proc. Roy. Soc. A, 233 (1955), 126-46.

8. - The characters of the cubic surface group, Proc. Roy. Soc. A, 237 (1956), 132-47.

9. J. S. Frame, The simple group of order 25920 , Duke Math. J., 2 (1936), 477-84.

10. - The classes and representations of the groups of 27 lines and 28 bitangents, Ann. di Mat. pura ed applicata, 32 (1951), 83-119.

11. A. F. Horadam, A locus in [8] invariant under a group of order $51840 \times 81$, Quart. J. Math. (Oxford) (2) 8 (1957) 241-59.

12. J. A. Todd, On the simple group of order 25920, Proc. Roy. Soc. A, 189 (1947), 326-58.

The University of New England 